\title{
A novel construct of anhedonia revealed in a Chinese sample via the Revised Physical and Social Anhedonia Scales
}

Qiongqiong $\mathrm{Wu}^{1+}$, Jiayue $\mathrm{He}^{1+}$, Shulin Fang ${ }^{1}$, Panwen Zhang ${ }^{1}$, Xingwei $\mathrm{Luo}^{1}$, Jianghua Zhang ${ }^{2}$, Yan Xiong ${ }^{2}$, Fusheng Luo ${ }^{3}$, Xiaosheng Wang ${ }^{4}$, Shuqiao Yao ${ }^{1,5,6}$ and Xiang Wang ${ }^{1,5,6^{*}}$ (D)

\begin{abstract}
Background: Anhedonia is a core clinical symptom of mental disorders. The Revised Physical Anhedonia Scale (RPAS) and the Revised Social Anhedonia Scale (RSAS) have been applied in clinical and non-clinical samples since 1980s. However, the construct of a unified RPAS\&RSAS for comprehensive measurement of anhedonia has never been explored. Therefore, the purpose of our study was to examine the factor structure of the unified RPAS\&RSAS among undergraduates and clinical patients.
\end{abstract}

Methods: A total of 3435 undergraduates from two universities and 294 clinical patients with mental disorders had completed the Chinese version of the RPAS and the RSAS. Exploratory factor analysis (EFA) and confirmatory factor analysis (CFA) were each conducted to reveal the constructs of the RPAS and the RSAS. CFA was used to evaluate first- and second-order models for the unified RPAS\&RSAS in undergraduates and clinical patients. The internal consistency and test-retest reliability of the RPAS and the RSAS were also evaluated.

Results: EFA and CFA indicated 2-factor structures for RPAS and RSAS, with the factors being defined as anticipatory anhedonia and consummatory anhedonia. The second-order model of the unified RPAS\&RSAS in the undergraduates and clinical patients both had satisfactory fit index values (Undergraduate sample: $C F I=0.901, T L I=0.899$, RMSEA $=$ $0.055, \mathrm{SRMR}=0.086$; Clinical sample: $\mathrm{CFI}=0.922, \mathrm{TLI}=0.911, \mathrm{RMSEA}=0.052, \mathrm{SRMR}=0.078$ ). The psychometric robustness of the RPAS\&RSAS were confirmed by high internal consistency and test-retest reliability values.

Conclusions: The unified RPAS\&RSAS with a second-order structure was confirmed in both undergraduates and clinical samples in Chinese. The construct of anhedonia was refreshed as covering physical and social domains, and each of them includes both anticipatory and consummatory components.

Keywords: Anhedonia, Revised Physical Anhedonia Scale (RPAS), Revised Social Anhedonia Scale (RSAS) , Factor structure, Second-order hierarchical model, Chinese version

\footnotetext{
*Correspondence: wang0916xia@gmail.com; wangxiang0916@csu.edu.cn

${ }^{\dagger}$ Qiongqiong Wu and Jiayue He contributed equally to this work.

${ }^{1}$ Medical Psychological Center, the Second Xiangya Hospital, Central South University, Changsha 410011, China

${ }^{5}$ Medical Psychological Institute of Central South University, Changsha 410011, China

Full list of author information is available at the end of the article
}

(c) The Author(s). 2020 Open Access This article is licensed under a Creative Commons Attribution 4.0 International License, which permits use, sharing, adaptation, distribution and reproduction in any medium or format, as long as you give appropriate credit to the original author(s) and the source, provide a link to the Creative Commons licence, and indicate if changes were made. The images or other third party material in this article are included in the article's Creative Commons licence, unless indicated otherwise in a credit line to the material. If material is not included in the article's Creative Commons licence and your intended use is not permitted by statutory regulation or exceeds the permitted use, you will need to obtain permission directly from the copyright holder. To view a copy of this licence, visit http://creativecommons.org/licenses/by/4.0/. The Creative Commons Public Domain Dedication waiver (http://creativecommons.org/publicdomain/zero/1.0/) applies to the data made available in this article, unless otherwise stated in a credit line to the data. 


\section{Background}

Anhedonia, defined as a diminished or absent ability to experience pleasure in normally pleasurable daily activities [1], has been associated with a neurotransmitter imbalance in reward processing circuitry involving multiple neuroanatomical areas [2-4] and dopamine dysregulation $[3,5,6]$. Conceptually, anhedonia has been suggested to be composed of two major components, namely anticipatory anhedonia and consummatory anhedonia [7]. Anticipatory pleasure is generated by reward motivation and behaviors aimed at future rewards (i.e., "wanting") while consummatory pleasure refers to pleasure in the present moment and reward attainment (i.e., "liking") [7]. Furthermore, results obtained from the Research Domain Criteria project, whose aim is to integrate information from genomics and circuits to behavior and self-reports in psychiatric disorders [8, 9], suggested that anhedonia may involve two domains, a Negative Valence Systems domain and a Social Processes domain (see www.nimh.nih.gov/research-priorities/rdoc/ index.shtml).

Anhedonia is experienced as a symptom of a number of mental disorders $[10,11]$, including major depressive disorder [12-16], post-traumatic stress disorder [11], obsessive-compulsive disorder [17], schizoid and schizotypal personality disorder $[18,19]$, and schizophrenia (as a negative symptom) [1, 20-23]. Therefore, there is a need for assessment and differential diagnosis with instruments that are not specific to a particular diagnosis.

The Chapman psychosis-proneness scales are series of scales that were designed to assess schizotypy tendencies in psychiatrically normal people [19, 24-27]. Four of the scales in this series, namely the Perceptual Aberration Scale, Magical Ideation Scale, Physical Anhedonia Scale, and Social Anhedonia Scale, form a schizotypy assessment battery known as the Wisconsin schizotypy scales (WSS). In recent years, the revised versions of the two anhedonia scales within the WSS, known as the Revised Physical Anhedonia Scale (RPAS) and the Revised Social Anhedo'nia Scale (RSAS), have been used alone across different cultures [28-31] and in various kinds of sample populations $[19,32,33]$. The RPAS assesses the lack of pleasure experienced in physical sensations, whereas the RSAS assesses the lack of pleasure in the social realm [34].

The RPAS and the RSAS are broadly appreciated for the inclusive information they cover, and their simple format, which requires participants to answer true/false questions. Testing of the psychometric robustness of the RSAS and RPAS has shown they both have good reliability (RPAS $\alpha=0.71-0.93$, RSAS $\alpha=0.82-0.95 ; \mathrm{r}_{\text {test-retest }}=$ $0.74-0.87)$ and good validity in the USA $[1,35]$, France [36], Germany [37, 38], Turkey [31], Spain [29] and China [30] (see Table 1). Although earlier the RSAS/ RPAS studies involved primarily clinical samples, recent studies have focused on non-clinical populations [39]. For example, Chan et al. showed that the RPAS and RSAS, which are self-report scales used to assess anhedonia severity across multiple neuropsychiatric stages [40], could be used to examine trait anhedonia in a nonclinical sample (i.e. college students) [30, 41]. The RSAS and RPAS have been used principally to assess schizotypy rather than anhedonia per se, and most previous studies have not included a large number of participants $[30,40,41]$. Thus, questions remain regarding the applicability of the RSAS and the RPAS, particularly with Chinese respondents.

Although the factor structures of Chapman's physical and social anhedonia scales have long been considered unidimensional, as was the intention when they were developed $[1,42]$, and that single dimension has been classified as a negative dimension of schizotypy in the context of Meehl's model [29], emerging evidence indicates that the RPAS and RSAS may have more complicated structures [43, 44]. The structure of the RPAS alone has not yet been reported, and that of the RSAS remains controversial, with studies reporting one [29, 43], two [45], and four [44] factor model fits, depending on language and subject sample. The two factors of the 2-factor RSAS model were defined as social apathy/aversion and social withdrawal, which are associated with the symptoms of the schizophrenia [44]. When the RSAS was loaded in both positive and negative WSS factors, model fitness outcomes were better than when RSAS was loaded in a purely negative factor [40, 46-48]. Although the two components of anhedonia (anticipatory and consummatory) have been distinguished in behavioral and psychometric studies [49], it remains to be clarified whether the anticipatory and consummatory anhedonia components exist in both the physical and social fields, and whether there is a hierarchical relationship between them [50]. Besides, although the RPAS and RSAS have been used together across different cultures [29, 30, 51], they have never been recognized as a combined unified scale (referred to as the RPAS\&RSAS from here forward), and the factor structure of the RPAS\&RSAS remains unrevealed.

The RPAS and RSAS, generally considered to be traditional anhedonia scales, were designed for patients with schizophrenia [1] and have been used extensively to assess anhedonia in schizophrenia studies [52]. Their appropriateness in other populations is unclear and has not been validated empirically [16]. Thus, there is a need to investigate their reliability and validity in other patient populations and non-clinical samples. The aim of this study was to analyze the individual structures of the RPAS and RSAS separately and the RPAS\&RSAS as a combined unified scale to explore the underlying construct of anhedonia both in clinical patient and 


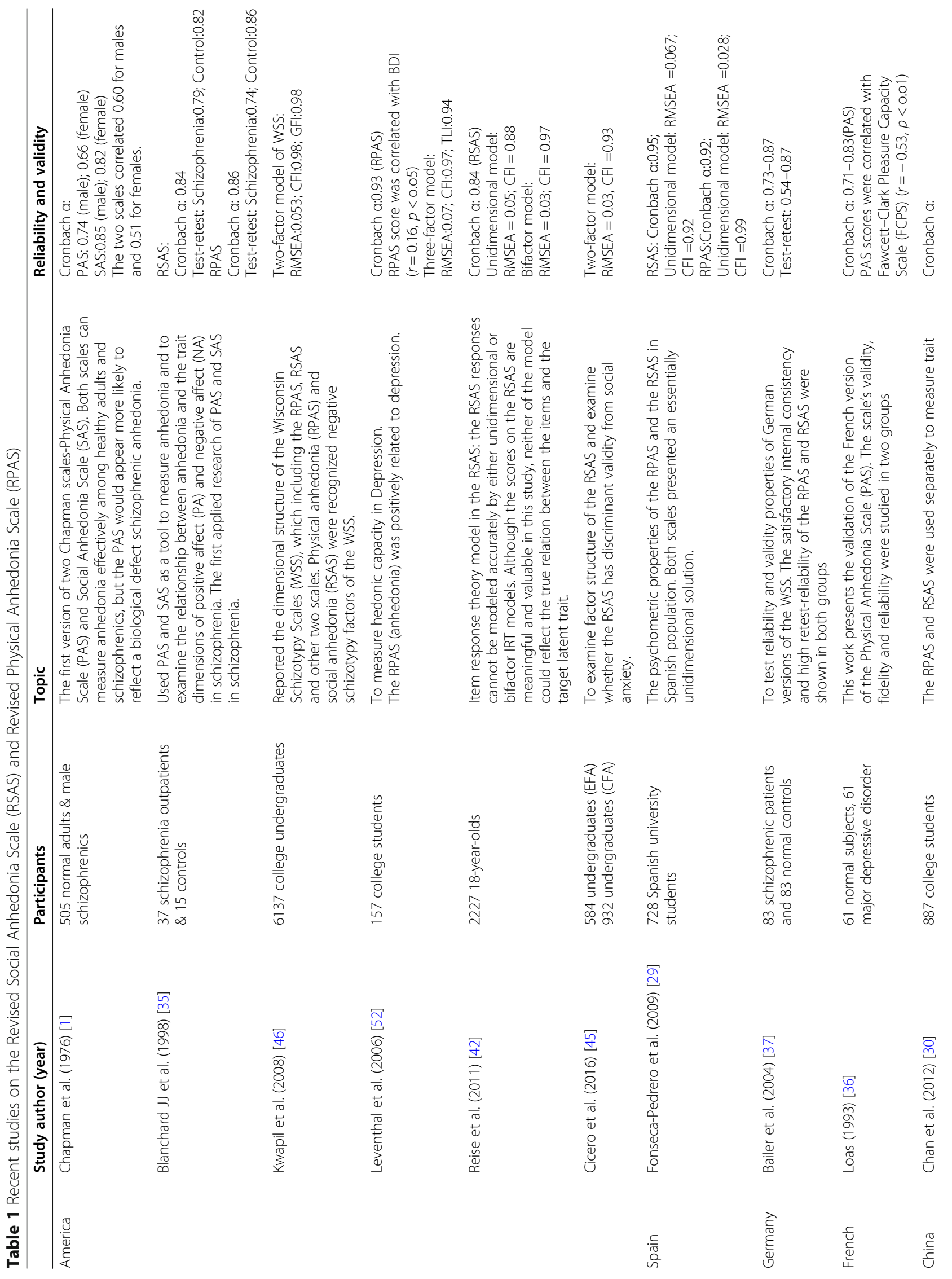




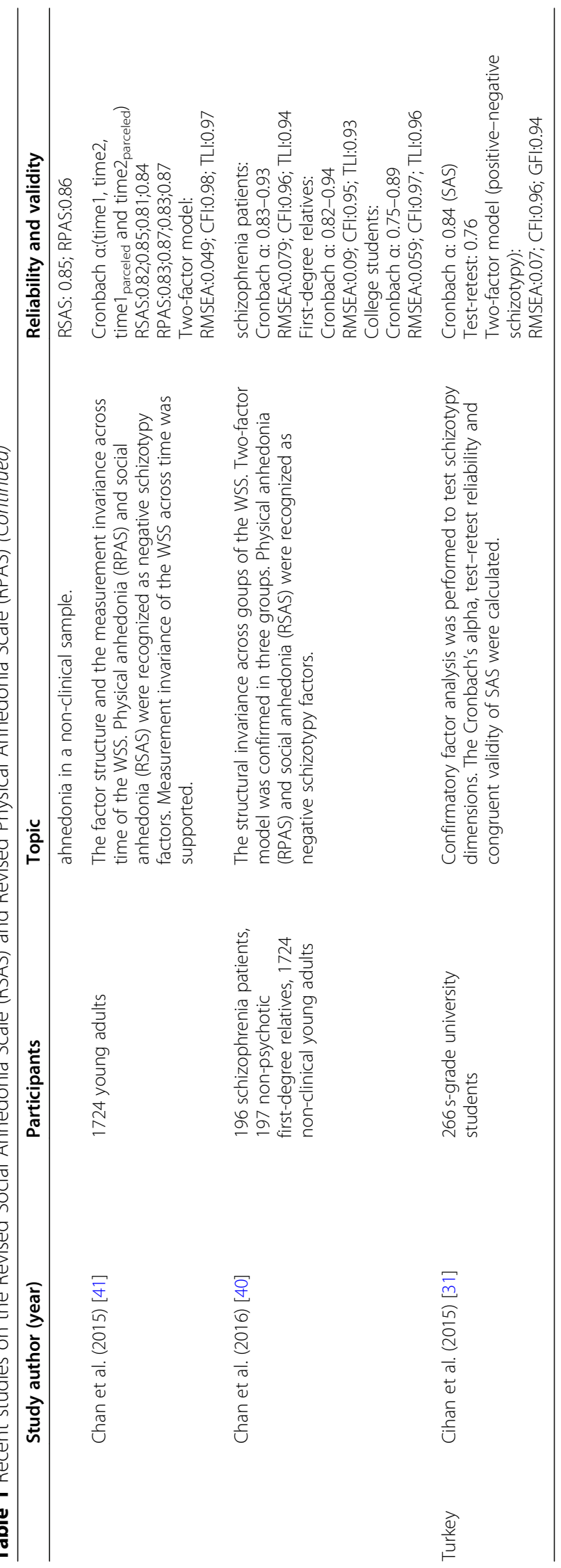


undergraduate samples. Firstly, we used Exploratory Factor Analysis (EFA) to explore the structure of the RPAS and RSAS in undergraduate samples. Secondly, we used confirmatory Factor Analysis (CFA) to confirm the structures of the RPAS and RSAS both in clinical patient and undergraduate samples. Third, we analyzed the structure of the unified RPAS\&RSAS for how it fits into a potential hierarchical model in clinical and undergraduate samples. The results obtained may be used to broaden the application of the RPAS and RSAS as measures of anhedonia, including in the context of various neuropsychiatric conditions, including anxiety and depression.

\section{Methods}

\section{Participants}

The sample size was calculated using the criteria of subject-to-item ratio of 10:1 or more [53], which giving a 1010 required sample size for EFA or CFA analysis. Based on the required sample size, this study recruited undergraduate sample from two Chinese universities in Hunan Province and they finished the scales in classroom. A total of 3537 university students completed the survey and $102(2.8 \%)$ subjects were excluded. So 3435 subjects with full data were left, including 1633 males and 1802 females, with mean ages \pm standard deviations of $18.37 \pm 0.23$ years and $18.11 \pm 0.28$ years, respectively. We assessed the mental disorders, a family history of any mental disorder, and physical disorders by selfreporting using a self-made questionnaire. The exclusion criteria were: (1) history of mental disorder, (2) family history of mental disorder (3) history of neurological disorder, (4) intellectual disability. To estimate test-retest reliability, we randomly selected 10 classes of students as a subgroup who had been completed the RPAS and RSAS twice with a one-month interval. Finally 223 students completed the retest questionnaire. The students did not responded anonymously and the tests were unpaid, and all the students volunteered to complete the test. If any student didn't want to participate, they could refuse to fill it out or drop out at any time.

The criteria of sample size is at least reach minimum sample size of 200 [54], after that, we try to meet the criteria of subject-to-item ratio of 10:1 [53]. In this study, the clinical samples reached the previous criteria, while the college student samples met both criteria. For the clinical sample, 348 clinical patients who had been referred for assessment and treatment in the psychological clinic of Second Xiangya Hospital were recruited. As subject-to-item ratio of 10:1 was not satisfied in the patient sample, the minimum sample size of 200 which was also considered in student sample was used in clinical patients. Finally, a total of 294 patients finished the questions, including 146 men (50\%) and 148 women $(50 \%)$, aged 16 to 37 years old (Mean $=24$; standard deviation $(\mathrm{SD})=15.9)$. The clinical sample only included psychiatric disorders which were ever reported to be correlated to anhedonia, such as major depressive disorder, obsessive-compulsive disorder, schizophrenia and the related personality disorder (depressive personality disorder, obsessive-compulsive personality disorder and schizotypal personality disorder). There was a significant age difference between the undergraduate sample and the clinical sample, the clinical sample was significantly older than the undergraduate sample $(\mathrm{t}=5.831, p<$ 0.001 ), but no significant gender difference was found between the two samples $\left(x^{2}=0.841, \mathrm{df}=1, p=0.361\right)$. The score of the Beck Depression Inventory (BDI), the Beck Anxiety Inventory (BAI) and the Childhood Trauma Questionnaire (CTQ) of clinical patients are significantly higher than those of undergraduate sample $(p<0.001)$. Moreover, the PRAS, the RSAS and the RPAS\&RSAS score of clinical patients are also significant higher than undergraduate sample $(p<0.001)$. The socio-demographic of the two samples in detail are presented in Table 2.

Participants were told that the information in these scales would not be disclosed to anyone outside of the

Table 2 Socio-demographic characteristics of samples

\begin{tabular}{|c|c|c|c|c|c|c|}
\hline Characteristic description & & $\begin{array}{l}\text { Undergraduates } \\
\text { Sample }(n=3435)\end{array}$ & $\begin{array}{l}\text { Clinical } \\
\text { Sample }(n=294)\end{array}$ & Chi-Square/t & $p$ & Cohen's d \\
\hline Gender, n & Male/female & $1633 / 1802$ & $146 / 148$ & 0.841 & 0.361 & - \\
\hline Age, Mean \pm SD & Years & $18.73 \pm 0.78$ & $24.02 \pm 7.52$ & 5.831 & $<0.001$ & - \\
\hline \multirow[t]{6}{*}{ Scale, Mean \pm SD } & BDI & $6.51 \pm 6.91$ & $12.44 \pm 8.88$ & -11.22 & $<0.001$ & 0.73 \\
\hline & BAl & $26.71 \pm 6.76$ & $30.74 \pm 7.95$ & -7.78 & $<0.001$ & 0.54 \\
\hline & CTQ & $47.19 \pm 7.95$ & $49.94 \pm 14.30$ & -4.33 & $<0.001$ & 0.22 \\
\hline & RPAS & $17.33 \pm 7.61$ & $20.32 \pm 9.31$ & -6.58 & $<0.001$ & 0.34 \\
\hline & RSAS & $9.06 \pm 5.70$ & $15.41 \pm 7.96$ & -18.53 & $<0.001$ & 0.89 \\
\hline & RPAS\&RSAS & $22.46 \pm 11.50$ & $35.69 \pm 15.31$ & -13.40 & $<0.001$ & 0.95 \\
\hline
\end{tabular}


research team and written informed consent forms were completed by all participants. This study was approved by the ethics committee of Second Xiangya Hospital, Central South University.

\section{Materials \\ Revised physical Anhedonia scale (RPAS) and social Anhedonia scales (RSAS)}

Chinese versions of the RPAS and RSAS that were translated by linguists and psychologists from English into Chinese were used [34]. The RPAS focuses on typically pleasurable physical stimuli (e.g. food), whereas the RSAS assesses anhedonia related social stimuli and connection with others. The RPAS and RSAS contain 61 and 40 true-false items, respectively. The items are scored relative to standard answers. According to the standard answer, items that need reverse scored are 'False', and items that do not need reverse scored are 'True'. If the response to an item matches the item's standard answer, it is scored as a "1"; otherwise, it is scored as a "0". Higher scores are indicative of more severe anhedonia and elevated risk of mental disorders. Both the original English RPAS/RSAS $(\alpha=0.74 / 0.85)$ and the Chinese RPAS/RSAS (Cronbach's $\alpha=0.75 / 0.94$ ) have good internal consistencies (see Table 1).

\section{Beck depression inventory (BDI)}

The BDI is a multiple-choice self-reporting 21-item scale [55] used primarily to assess the presence and severity of depressive symptoms in the prior 2 weeks in clinical and non-clinical populations (e.g. guilty feelings; loss of pleasure). Each question is answered on a 0-3-point scale of intensity. The BDI total score range is from 0 to 63 points, with higher scores indicating more severe symptoms. In this study, the Cronbach's $\alpha$ of BDI was 0.85 in undergraduate sample and 0.84 in clinical sample.

\section{Beck anxiety inventory (BAl)}

The BAI includes 21 items [56] that assess the degree to which subjects are disturbed by various anxiety symptoms (e.g. Unable to relax; Nervous). Each item is scored on a 4-point scale, ranging from 1 (not at all) to 4 (severely). The total score ranges from 21 to 84 . In this study, the Cronbach's $\alpha$ of BAI was 0.90 in undergraduate sample and 0.83 in clinical sample.

\section{Childhood trauma questionnaire (CTQ)}

The CTQ is a 28-item [57] retrospective self-report questionnaire to assess childhood trauma experience before the age of 16. It has five subscales: emotional neglect (e.g., "felt loved,"), physical neglect (e.g., "was taken to the doctor,"), emotional abuse (e.g., "felt emotional abused"), sexual abuse (e.g., "was molested,") and physical abuse (e.g., "was hit so hard by family"). Five items each assess all five types of maltreatment, another 3 items was taken as validity evaluation (e.g., "perfect childhood"). Each item is scored on a 5-point scale. Each subscale was varied from 5 to 25 scores and the total score was in the range from 25 to 125 score. In this study, the Cronbach's $\alpha$ of CTQ was 0.72 in undergraduate sample and 0.68 in clinical sample.

\section{Data analysis}

IBM SPSS Statistics 20.0 was used for descriptive statistics and M-plus 7.11 software was used for factor analysis (EFA and CFA). Data from participants with missing data and participants that met the exclusion criteria were excluded. Mean descriptive statistic values are reported with 95\% confidence intervals (CIs). Cronbach's $\alpha$ values were calculated to evaluate reliability (i.e. internal consistency). A minimum standard of 0.70 is set for Cronbach's $\alpha$ coefficients, but an $\alpha$ of 0.60 is also considered acceptable. Test-retest reliability was assessed with Pearson correlation analyses. Some items (physical, $1 / 4 / 9 / 40 / 53$, and social 33) with low relevance or inappropriate meaning were excluded from the Chinese RPAS and RSAS to make them more suitable for Chinese youth. The excluded items had Pearson $r$ values < 0.100 , indicating very weak associations with pleasure capacity in Chinese youth. Additionally, four of the excluded physical items $(1,9,40$, and 53$)$ address sexual issues; these items can be excluded when applied to teenagers with limited sexual experience .

In the undergraduate sample, we split the collected statistics in half randomly. There is no difference in age and gender between two samples. First, the EFA was conducted on a randomly split-half of the whole sample $(n=1770)$. EFA was used to identify the best fitting factor model of the RPAS and RSAS, in the present sample. Subsequently, we used a random split-half $(n=1769)$ of the sample to run CFA. The CFA tested the fit of the model that was generated from our EFA. Finally, utilizing the full sample we conducted CFA to estimate the factor structure of the simplified unified RPAS\&RSAS. Besides, we also conducted the CFA to estimate the structure of the RPAS, the RSAS and the unified RPAS\&RSAS among clinical patients.

In the EFA, categorical variables were analyzed with the mean and variance adjusted (WLSMV) estimator, the most common and effective analysis method for categorical variables [58], in the M-plus program. Parceling was not performed in the EFA because the scale structures were unknown.

In preparation for CFA, items were parceled into individual factors according to the internal consistency approach for multidimensional scales to simplify the unified RPAS\&RSAS. A random algorithm was used in 
the parceling for its convenience and validity. For fouritem parceled items, mean parcel scores were used as final scores, thereby changing categorical variables into quantitative variables. CFA of the simplified unified RPAS\&RSAS was thus conducted with a maximum likelihood (ML) estimator for the first-order (physical anhedonia and social anhedonia) and second-order (anticipatory physical/social anhedonia and consummatory physical/social anhedonia) models. The TuckerLewis index (TLI), comparative fit index (CFI), standard root mean square residuals (SRMR), and root mean square error of approximation (RMSEA) methods were used to determine goodness of fit [59]. The criteria for accepting the model were: TLI $\geq 0.900, C F I \geq 0.900$, SRMR $\leq 0.080$, and RMSEA $\leq 0.080[60,61]$.

\section{Results}

\section{Descriptive statistics}

In the undergraduate sample, 32 students were removed from the sample due to being outliers, total scores for the uniformed RPAS\&RSAS ranged from 2 to 61 $($ Mean $=22.46 ; \mathrm{SD}=11.50)$. The skewness for each item ranged from .04 to 3.65 , kurtosis ranged from .01 to 3.66 (Table S1 in supplementary material).

In clinical sample, total scores for the uniformed RPAS\&RSAS ranged from 7 to 80 (Mean $=35.69 ; \mathrm{SD}=$ 15.31). The skewness for each item ranged from .02 to 2.65, kurtosis ranged from .01 to 2.83 (Table S2 in supplementary material).

\section{Reliability: internal consistency and test-retest reliability} The Cronbach's $\alpha$ coefficient of the RPAS\&RSAS were 0.884 and 0.672 in the undergraduates and the clinical patients respectively, which indicated good reliability with respect to internal consistency. The Pearson's $r$ values obtained for the RPAS\&RSAS 0.644 $(p<0.001)$ in a subsample of 223 participants, indicating good testretest reliability.

\section{Construct validity: RPAS/RSAS factor analysis}

After the elimination of six items for culture inappropriateness or poor relevance (see Methods), EFA of the RPAS (56 items) and RSAS (39 items), involving half of the participants, was conducted with a WLSMV estimator. The model fit indexes obtained for the RPAS and RSAS are presented in Table 3 and Table 4, respectively. As illustrated in Table 3, only the 4-factor model meet the criteria for accepting the model $(\mathrm{CFI}=0.924$, $\mathrm{TLI}=0.912$, SRMR $=0.053$, RMSEA $=0.024$ ). Thus, the 4-factor model was the best fitting factor structure of the RPAS. The RSAS fit well with a 2-factor structure model $\quad(\mathrm{CFI}=0.941, \quad \mathrm{TLI}=0.934, \quad \mathrm{SRMR}=0.063$, RMSEA $=0.028)$ as shown in Table 4 . The factor loadings of each item are reported in Table S3 and Table S4 in the supplementary material.

In the RPAS, the physical anhedonia items segregated into four factors as follows: Factor 1 contains items 5, 6, $8,10,11,12,13,14,16,17,18,20,22,23,26,27,28,29$, $32,33,43,44,48,50,51,52,55,56$, and 57; Factor 2 contains items_2, 3, 19, 21, 24, 25, 30, 31, 34, 35, 36, 37, $39,41,45,46,47,49,54,58,59,60$, and 61; Factor 3 contains items 7 and 15; and Factor 4 contains items 38 and 42. However, as can be seen in Table S4, Factor 3 and Factor 4 each have only two items, and all four of these items had relatively high loadings (loadings $>0.350$ [62]) in the first two factors (item 15 loaded 0.448 in Factor 1; item 7, 38, 42 loaded 0.389, 0.471 and 0.363 in Factor 2 respectively). Thus, we spread these four items into the first two factors according to loading parameters. Accordingly, item 15 was placed with Factor 1 and items 7, 38, and 42 were placed with Factor 2.

The RPAS items gathered in Factor 1 (P1) were related to consummatory physical pleasure whereas the RPAS items gathered in Factor 2 (P2) were related to anticipatory physical pleasure. Regarding items gathered in Factors 3 and 4, the content of item 15 (There aren't many things I really like to do) associated well with "liking", thus relating it to consummatory physical anhedonia (Factor 1). In contrast, the content of items 7 (The taste of food has always been important tome), 38 (The beautiful scenery can make me feel delighted). and 42 (I seldom have the idea of singing in the bath) associated well with "wanting", thus relating them to anticipatory physical anhedonia (Factor 2).

The RSAS items gathered in Factor 1 (S1) -that is, items $1,2,3,6,10,13,14,17,21,22,23,26,27,28,29$, $32,34,35,37,38,39$, and 40 -were related to the consummatory social pleasure. The RSAS items gathered in

Table 3 The fitness indicators in the EFA of the models of the RPAS

\begin{tabular}{lllllll}
\hline Model & Chi-Square & df & CFI & TLI & SRMR & RMSEA(90\%Cl) \\
\hline 1-factor & 7866.714 & 1430 & 0.625 & 0.611 & 0.133 & $0.051(0.050,0.052)$ \\
2-factor & 4036.320 & 1367 & 0.845 & 0.833 & 0.073 & $0.034(0.032,0.035)$ \\
3-factor & 3226.631 & 1375 & 0.897 & 0.884 & 0.060 & $0.028(0.027,0.029)$ \\
4-factor (modified 2-factor) & 2567.392 & 1271 & 0.924 & 0.912 & 0.053 & $0.024(0.023,0.026)$ \\
\hline
\end{tabular}

RPAS revised physical anhedonia scale, $d f$ degree of freedom, CFI comparative fit index, TLI tucker lewis index, SRMR standard root mean square residuals, RMSEA root mean square error of approximation, $90 \% \mathrm{Cl} 90 \%$ confidence Interval 
Table 4 The fitness indicators in the EFA of the models of the RSAS

\begin{tabular}{lllllll}
\hline Model & Chi-Square & df & CFI & TLI & SRMR & RMSEA(90\% CI) \\
\hline 1-factor & 4436.681 & 702 & 0.756 & 0.742 & 0.130 & $0.056(0.054,0.057)$ \\
2-factor & 1569.098 & 664 & 0.941 & 0.934 & 0.063 & $0.028(0.026,0.030)$ \\
\hline
\end{tabular}

RSAS revised social anhedonia scale

Factor 2 (S2)-that is, items 4, 5, 7, 8, 9, 11, 12, 15, 16, $18,19,20,24,25,30,31$, and 36-were related to anticipatory social pleasure.

For CFA of two scales in undergraduate samples, we parceled randomized four items and simplified the scales because the RPAS/RSAS items seemed excessive and scattered. There were 14 parcels in the RPAS (7 in P1 and 7 in P2) and 9 parcels in the RSAS (5 in S1 and 4 in S2). As is shown in Table 5, fit index values supported a good fit with a 2 -factor model for the RPAS $(C F I=0.947, \quad$ TLI $=0.936, \quad$ SRMR $=0.039$, RMSEA $=0.052$ ) and a good fit with a 2-factor model for the RSAS (CFI $=0.967$, TLI $=0.957$, SRMR $=0.037$, RMSEA $=0.048$ ) in CFA of the undergraduates, consistent with our EFA results. In the clinical sample, the results of CFA corresponded with the non-clinical sample's and supported 2-factor models for both the RPAS and the RSAS (RPAS: CFI $=0.947$, TLI $=0.932$, SRMR $=0.044, \quad$ RMSEA $=0.052 ; \quad$ RSAS: CFI $=0.977$, $\mathrm{TLI}=0.968, \mathrm{SRMR}=0.041$, RMSEA $=0.061)$.

\section{Factor analysis of the RPAS\&RSAS}

Based on the good fits of the 2-factor models for the two individual scales and good first-order model fitness results for the RPAS\&RSAS (Table 6), CFA confirmed a novel second-order model for the RPAS\&RSAS as a unified scale. The second-order model were good fitted in clinical sample: $\mathrm{CFI}=0.922$, $\mathrm{TLI}=0.911$, $\mathrm{SRMR}=0.078$, RMSEA =0.052). But in undergraduate sample the second-order model was good fitted overall (CFI $=0.901$, $\mathrm{TLI}=0.899, \mathrm{SRMR}=0.086$, $\mathrm{RMSEA}=0.055$ ) except that SRMR was slightly missed the margin of criteria for acceptable fit. The model fit indexes supported a simpler construct of anhedonia, as shown in Fig. 1. The results supported that with a lower number of degrees of freedom and a more simple structure, the second-order

Table 5 The fitness indicators the CFA and the models of the RPAS and the RSAS

\begin{tabular}{lllllll}
\hline \multicolumn{2}{c}{ Chi-Square } & df & CFI & TLI & SRMR & RMSEA(90\%CI) \\
\hline \multicolumn{2}{l}{ Undergraduates } \\
RPAS & 445.376 & 76 & 0.947 & 0.936 & 0.039 & $0.052(0.048,0.057)$ \\
RSAS & 171.468 & 34 & 0.967 & 0.957 & 0.037 & $0.048(0.041,0.055)$ \\
$\begin{array}{l}\text { Clinical patients } \\
\text { RPAS }\end{array}$ & & & & & \\
RSAS & 53.231 & 26 & 0.977 & 0.968 & 0.041 & $0.061(0.037,0.084)$ \\
\hline
\end{tabular}

model showed unique advantage in hierarchy to support the perspective of multiple dimensions in anhedonia, which combines observable behavioral symptoms and underlying biological mechanisms.

\section{Discussion}

The current study examined the psychometric properties of a unified RPAS\&RSAS as a distinct anhedonia assessment tool in a large sample of young Chinese adults and clinical patients for the first time. The results not only showed that good reliabilities and validities of RPAS\&RSAS, but also supported an underlying hierarchical structure of anhedonia. Specifically, we accepted a simple and accurate second-order model of the RPAS\&RSAS, which was confirmed to have a good fit in clinical patients, supporting the robust construct validity of the combined unified scale. As anhedonia is composed of consummatory anhedonia and anticipatory anhedonia from the reward processing models in the neurobiology, the construct of RPAS\&RSAS (the "gold standard" for measuring hedonic capacity) is of great possibility to be consistent with the components of anhedonia.

Initially, anhedonia was considered as a psychopathological symptom among clinical patients. According to external behavior of patients, phenomenological researches suggested anhedonia had two major components, including physical anhedonia and social anhedonia [29]. In the past two decades, with further researches on the genetic, phenomenological, and molecular mechanisms of it, anhedonia was more and more considered as a multidimentional concept that encompasses physical/social dimension, consummatory/anticipatory dimension, and so on [30]. The current study combined the RPAS and RSAS as a whole to assess its psychometric properties in both clinical and non-clinical samples, and further explored the multiple conceptual dimensions of anhedonia contained in this scale. The results showed that it was feasible and suitable to use a unified RPAS\&RSAS instrument as a novel distinct instrument, in which the acceptable Cronbach's $\alpha$ values supported good internal consistency for anhedonia (physical and social), and the high RPAS\&RSAS testretest reliability coefficient indicated the temporal stability of anhedonia. Additionally, a good fitting yielded by the second-order model in this study provided new evidence to the multi-dimensionality of anhedonia. It should be noted that a previous study showed that RPAS and RSAS may have high degree of cultural bias demonstrated from questions [16]. Further researches are needed to assess the measurement invariance of unified RPAS\&RSAS cross cultures.

With respect to the factorial validity, the RSAS was found to have a 2-factor structure both in undergraduates and clinical patients in this study, with the two 
Table 6 The fitness indicators in the CFA of the models of the RPAS\&RSAS

\begin{tabular}{|c|c|c|c|c|c|c|}
\hline & Chi-Square & df & CFI & TLI & SRMR & RMSEA(90\%Cl) \\
\hline \multicolumn{7}{|l|}{ Undergraduates } \\
\hline First-order model & 1628.053 & 224 & 0.941 & 0.934 & 0.038 & $0.042(0.040,0.044)$ \\
\hline Second-order model & 2592.801 & 225 & 0.901 & 0.899 & 0.086 & $0.055(0.053,0.056)$ \\
\hline \multicolumn{7}{|l|}{ Clinical patients } \\
\hline First-order model & 362.611 & 224 & 0.936 & 0.928 & 0.033 & $0.047(0.038,0.056)$ \\
\hline Second-order model & 390.599 & 222 & 0.922 & 0.911 & 0.078 & $0.052(0.044,0.061)$ \\
\hline
\end{tabular}

components being defined as consummatory and anticipatory social anhedonia. This 2-factor model is consistent with Cicero's et al.'s study [44], with only items 24 and 25 having different factor designation relative to the EFA results. Cicero defined their two RSAS factors as social apathy/aversion and social withdrawal based on an emotion and behavior perspective, and in the context of potential symptoms of schizophrenia [44]. According to the recent research progress on anhedonia, we proposed that the two factors explored by both studies may reflect the common underlying mechanisms of anhedonia prior described in numerous previous literature: the anticipatory and consummatory anhedonia [63]. Specifically, anticipatory social anhedonia, or social withdrawal defined by Cicero, is characterized by a loss of the motivation to connect to others; while consummatory social anhedonia, or social apathy/aversion, is characterized by a lack of emotional connection or involvement in social activities [7]. The terms consummatory social anhedonia and anticipatory social anhedonia can be related to potential biological mechanisms whereas the terms social withdrawal and social apathy are derived from schizotypy symptoms. It is hoped that the context and links made in this study may promote application of the RSAS for assessment of a broader range of mental disorders (beyond the schizophrenia spectrum) in the future $[7,13$, 49].

Similar to our findings with the RSAS, we also obtained a 2-factor structure for the RPAS: consummatory physical anhedonia and anticipatory physical anhedonia. We excluded items $1,4,9,40$, and 53 from the RPAS due to their low relevance, consistent with the previous study [54]. The RPAS has long been assigned to the negative schizotypy dimension in the WSS, in which its unidimensionality has been taken for granted [1, 64]. However, animal behavioral experiments in depression [65] and schizophrenia [66, 67] models suggested that physical anhedonia has more than one dimension. For example, the sucrose preference test relies on an affinity for a physical stimulus (consummatory pleasure) whereas the forced swimming test relates to "wanting" (anticipatory pleasure) [16]. Elucidation of the anhedonic capacity concept [7], including consummatory and anticipatory components, in the context of neurobiological arguments underscores an inherent complexity of the RPAS construct. Meanwhile, the potential utility for using the RPAS to assess anhedonia generally over the lifespan also points to its potential multi-dimensionality [52]. Finally, data from other measures support a psychometric multidimensionality of physical anhedonia. For example, some items in the Temporal Experience of Pleasure Scale involve the pleasure of tasting food and these aspects of pleasure were represented in the consummatory and anticipatory components of the RPAS [49], as indicated in our 2-factor model.

It is worthy to note that our study not only confirmed the 2-factor model including consummatory and anticipatory components in both RPAS and RSAS, but also proved a simple and accurate second-order model of the RPAS\&RSAS to further support the robust construct validity of the combined unified scale. In addition, that hierarchical model was confirmed to have a good fit in both the sample of undergraduate students and clinical patients. In fact, the notion of anhedonia as encompassing social and physical aspects was developed in the 1970s. There is a long history and broad acceptance of viewing mental disorders in relation to physical sensation and social function, and this perspective is well represented in diagnostic criteria. These bases are also amenable to experimental exploration of the nature of anhedonia in animal experiments [68, 69]. The recent theory of temporal components of anhedonia, namely anticipatory and consummatory anhedonia [7], were also supported by our hierarchical analysis of the concept of anhedonia.

In our model shown in Fig. 1, anhedonia is divided firstly into physical and social anhedonia and secondly each aspect of anhedonia is divided into anticipatory and consummatory components. The unified RPAS\&RSAS, separated from the WSS, has proven to be a scale of simple construction that integrates multiple levels of information related to anhedonia, which makes it a convenient and useful tool for researchers and clinical psychologists. Hedonic capacity has been related to genetics, neurochemical disorders, and specific brain regions $[2,50]$. From the transdiagnostic perspective, anhedonia is considered as the emotion and reward processing deficiency and reflects an endophenotype (i.e. an 


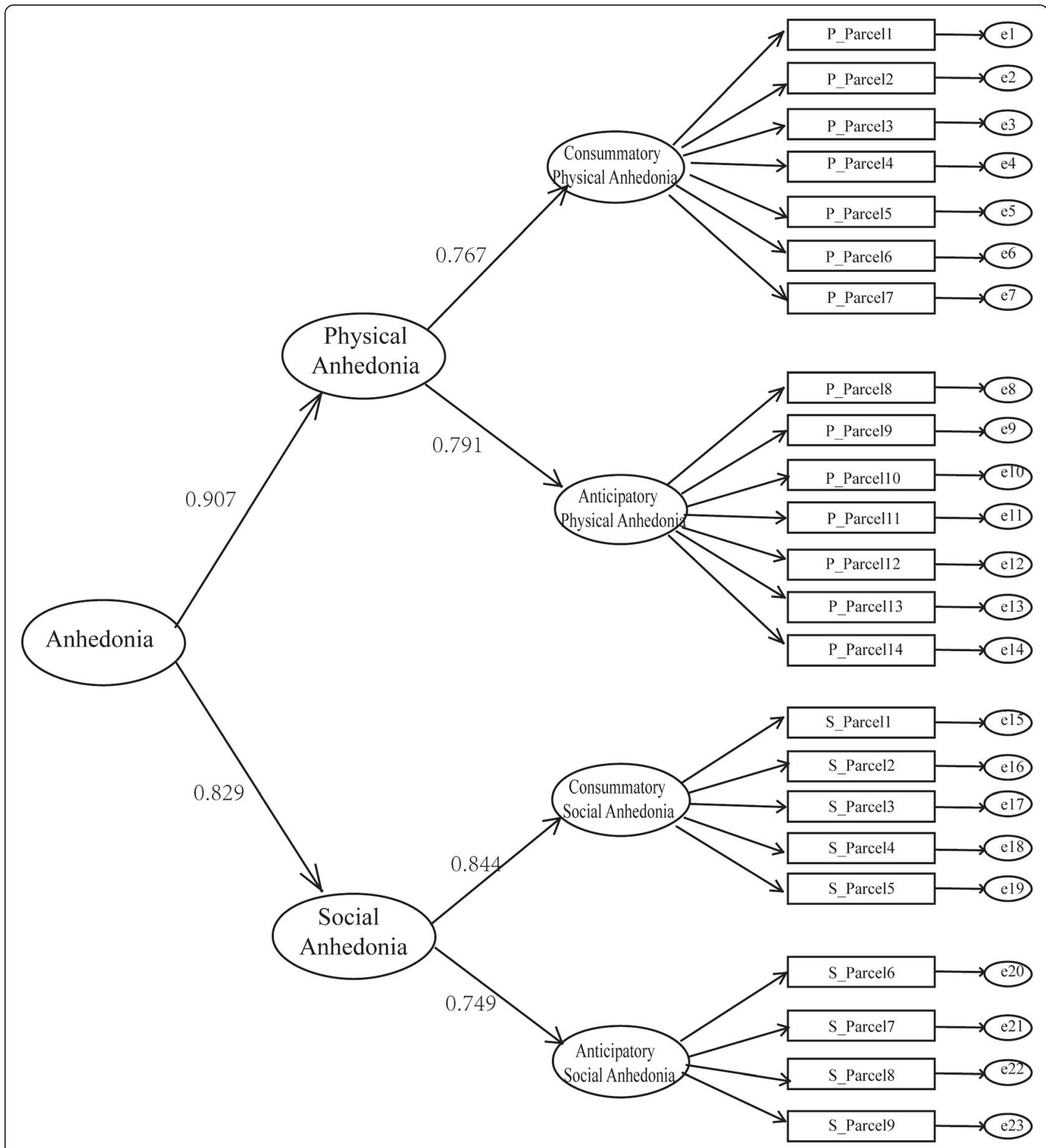

Fig. 1 Second-order model of the Revised Physical and Social Anhedonia Scales (RPAS and RSAS) and the underlying construct of anhedonia

intermediate phenotype of trait that is expressed along a spectrum), which can be a component of multiple mental disorders, including schizophrenia and depression $[12,70]$. Additionally, those neuropsychiatric deficit endophenotypes have been linked to genetic signatures and molecular mechanisms [71-73]. Thus, the secondorder structure found for the RPAS\&RSAS may help provide insight into the integration of multiple levels of information, including genetic, endophenotype, and symptom expression, in various mental disorders from the perspective of an anhedonia measure.

According to the previous literatures, anhedonia is a prominent symptom of several neuropsychiatric disorders, especially in major depressive disorder (MDD) 
and schizophrenia [13, 18]. Anhedonia is characterized as "loss of interest or pleasure in daily life" and recognized as one of two essential features of MDD $[74,75]$. Meanwhile, anhedonia is also the most common negative symptom of schizophrenia, manifested as apathy or indifference [18]. According to the National Institute of Mental Health (NIMH) Research Domain Criteria (RDoC) project, researchers trying to use the basic behavioral dimension of functioning to identify anhedonia in a transdiagnostic approach. Thus, a suitable tool is needed to measure the anhedonia comprehensively. Based on the latest studies, anhedonia is a multidimensional construct, such as physical/social, anticipatory/consummatory or motivation/experience dimension. In clinical observation, patients with mental illness show different patterns of composition in the subcomponent of anhedonia. For instance, patients with schizophrenia reported more remarked social anhedonia than physical anhedonia $[76,77]$. However, in patients with major depressive disorder, both significant physical anhedonia and social anhedonia were observed [78, 79]. Similarly, a number of studies showed that patients with different diagnoses had different impairments in anticipatory and consummatory aspects. A recent meta-analysis of anticipatory and consummatory pleasure in schizophrenia reported that anticipatory pleasure may be significantly impaired in patients compared to the consummatory pleasure [80]. An ALE meta-analysis also revealed that consummatory and anticipatory were associated with different neural mechanisms of anhedonia in MDD and schizophrenia [4]. Hence, exploring the construct of anhedonia and distinguishing the different subcomponents of anhedonia are essential for mental illnesses. However, there is no qualified assessment both covering the classification of physical and social anhedonia, as well as anticipatory and consummatory anhedonia, which is in need for exploring the anhedonia in various kinds of psychiatric disorders. Thus, this study confirmed the good reliabilities and validities of RPAS\&RSAS as a unified measurement and revealed a second-order model in both healthy and clinical samples, which provides new possibilities for measuring anhedonia in various mental disorders and help to promote more precise treatment approaches.

The RSAS and RPAS were comprehensive measurement tools for assessing anhedonia. Compare to rating scale, the RPAS\&RSAS was written in simple language with a yes/no response format, which makes it easy to understand and patients spend less time to finish [29]. Since its comprehensive and easy to understand, the RSAS and RPAS has been widely used in clinical patients. In this study, it only took about 10 min for the clinical patients to complete the combining scale.

Although the results of the present study demonstrated a stable second-order model and appropriate reliability scores for the Chinese version of the unified RPAS\&RSAS, several limitations should to be acknowledged. Firstly, reliance on an undergraduate student sample may limit the generalizability of our findings to individuals in other age bands. Secondly, our results are based on a cross-sectional study, which did not take into consideration changes that may occur over time within individuals. Further longitudinal studies should be conducted to investigate the psychometric properties of the unified RPAS\&RSAS in more age group.

\section{Conclusion}

In summary, the study explored the psychometric properties of the RPAS, the RSAS and the unified RPAS\&RSAS in the undergraduates and clinical patients. A second-order model of the RPAS\&RSAS was brought out for the first time and was selected over a first-order model for its ability to optimize simplicity and accuracy. The novel construct for anhedonia was further confirmed both in healthy undergraduate sample and clinical sample. Our study may promote the understanding of the multiple dimensions in anhedonia and broaden the application of the unified RPAS\&RSAS as an individual measurement in various psychiatric disorders.

\section{Supplementary information}

Supplementary information accompanies this paper at https://doi.org/10. 1186/s12888-020-02900-w.

Additional file 1.

\section{Abbreviations}

RPAS: Revised physical anhedonia scale; RSAS: Revised social anhedonia scale; RPAS\&RSAS: Unified, simplified RPAS and RSAS; EFA: Exploratory factor analysis; CFA: Confirmatory factor analysis; WSS: Wisconsin Schizotypy Scales

\section{Acknowledgements}

We would like to thank all leaders, teachers and working staff. Without their help, it would be much harder for us to finish this paper.

\section{Authors' contributions}

XW and SY supervised the study. QW and JH performed the analysis and wrote paper. SF, XL and PZ contributed to the analysis. JZ, YX and FL provided substantial modification to the manuscript. All co-authors revised and approved the version to be published.

\section{Funding}

This study was supported by Chinese Ministry of Education's Humanities and Social Science Research Project (Grant No. 13YJA190015) in the design of the study. This study was also supported by Education Reform Project of General Colleges and Universities in Hunan Province (Grant No. 2017jy77), National Natural Science Foundation of China (Grant No. 31671144), Shanghai Municipal Science and Technology Major Project (Grant No. 2018SHZDZX01) in data collection and analysis. Besides, the Hunan Provincial Natural Science Foundation of China (Grant No. 2019JJ40362) played a vital role in interpretation of data and in writing the manuscript. 


\section{Availability of data and materials}

The datasets generated and analysed during the current study are not publicly available due to no permission from participants to share anonymized participant data publicly but are available from the corresponding author on reasonable request.

\section{Ethics approval and consent to participate}

This study was approved by the ethics committee of Second Xiangya Hospital, Central South University. All participants were over 16 years old and had written informed consent.

\section{Consent for publication}

Not applicable.

\section{Competing interests}

The authors declare that they have no competing interests.

\section{Author details}

${ }^{1}$ Medical Psychological Center, the Second Xiangya Hospital, Central South University, Changsha 410011, China. ${ }^{2}$ Student Affairs Department, Central South University, Changsha 410083, China. ${ }^{3}$ Student Affairs Department, Central South University of Forestry and Technology, Changsha 410004 China. ${ }^{4}$ Department of Human Anatomy and Neurobiology, Xiangya School of Medicine, Central South University, Changsha 410013, China. ${ }^{5}$ Medical Psychological Institute of Central South University, Changsha 410011, China. ${ }^{6}$ National Clinical Research Center for Mental Disorders, Changsha 410011, China.

\section{Received: 18 April 2020 Accepted: 28 September 2020} Published online: 09 November 2020

\section{References}

1. Chapman L, Chapman JP, Raulin ML. Scales for physical and social anhedonia. J Abnorm Psychol. 1976;85(4):374-82.

2. Der-Avakian A, Markou A. The neurobiology of anhedonia and other reward-related deficits. Trends Neurosci. 2012:35(1):68-77.

3. Robbins TW. NEUROSCIENCE. Illuminating anhedonia. Science (New York). 2016;351(6268):24-5.

4. Zhang B, Lin P, Shi H, Ongur D, Auerbach RP, Wang X, Yao S, Wang X. Mapping anhedonia-specific dysfunction in a transdiagnostic approach: an ALE meta-analysis. Brain Imaging Behav. 2016;10(3):920-39.

5. Szczypinski JJ, Gola M. Dopamine dysregulation hypothesis: the common basis for motivational anhedonia in major depressive disorder and schizophrenia? Rev Neurosci. 2018;29(7):727-44.

6. Ettinger U, Mohr C, Gooding DC, Cohen AS, Rapp A, Haenschel C, Park S. Cognition and Brain Function in Schizotypy: A Selective Review. Schizophrenia Bull. 2015;41(suppl_2):S417-26.

7. Chen $Y, X u J$, Zhou L, Zheng Y. The time course of incentive processing in anticipatory and consummatory anhedonia. J Affect Disord. 2018:238:442-50.

8. Cuthbert BN. The RDoC framework: facilitating transition from ICD/DSM to dimensional approaches that integrate neuroscience and psychopathology. World Psychiatry. 2014;13(1):28-35.

9. Patrick CJ, Hajcak G. RDoC: translating promise into progress. Psychophysiology. 2016;53(3):415-24.

10. Loas G, Krystkowiak P, Godefroy O. Anhedonia in Parkinson's disease: an overview. J Neuropsychiatry Clin Neurosci. 2012;24(4):444-51.

11. Nawijn L, van Zuiden M, Frijling JL, Koch SB, Veltman DJ, Olff M. Reward functioning in PTSD: a systematic review exploring the mechanisms underlying anhedonia. Neurosci Biobehav Rev. 2015;51:189-204.

12. Pizzagalli DA. Depression, stress, and anhedonia: toward a synthesis and integrated model. Annu Rev Clin Psychol. 2014;10:393-423.

13. Treadway MT, Zald DH. Reconsidering anhedonia in depression: lessons from translational neuroscience. Nat Rev Neurosci. 2011;35(3):537-55.

14. McIntyre RS, Woldeyohannes HO, Soczynska JK, Maruschak NA, WiumAndersen IK, Vinberg M, Cha DS, Lee Y. Anhedonia and cognitive function in adults with MDD: results from the International Mood Disorders Collaborative Project. CNS Spectrums. 2016;21(5):362-6.

15. Chow TK, KS, Rizvi SJ. Anhedonia as a Crucial Factor of Depression: Assessment, Neurobiological Underpinnings and Treatment. Singapore: Springer; 2018.
16. Rizvi SJ, Pizzagalli DA, Sproule BA, Kennedy SH. Assessing anhedonia in depression: potentials and pitfalls. Neurosci Biobehav Rev. 2016;65:21-35.

17. Assogna F, Cravello L, Caltagirone C, Spalletta G. Anhedonia in Parkinson's disease: a systematic review of the literature. Mov Disord. 2011;26(10):1825-34.

18. Blanchard JJ, Collins LM, Aghevli M, Leung WW, Cohen AS. Social Anhedonia and Schizotypy in a community sample: the Maryland longitudinal study of Schizotypy. Schizophr Bull. 2009;37(3):587-602.

19. Fluckiger R, Ruhrmann S, Debbane M, Michel C, Hubl D, Schimmelmann BG, Klosterkotter J, Schultze-Lutter F. Psychosis-predictive value of self-reported schizotypy in a clinical high-risk sample. J Abnorm Psychol. 2016;125(7):923-32.

20. Marder SR, Galderisi S. The current conceptualization of negative symptoms in schizophrenia. World Psychiatry. 2017;16(1):14-24.

21. Kwapil TR. Social anhedonia as a predictor of the development of schizophrenia-spectrum disorders. J Abnorm Psychol. 1998;107(4):558-65.

22. Burbridge JA, Barch DM. Anhedonia and the experience of emotion in individuals with schizophrenia. J Abnorm Psychol. 2007;116(1):30-42.

23. Strauss GP, Gold JM. A new perspective on anhedonia in schizophrenia. Am J Psychiatry. 2012;169(4):364-73.

24. Chapman LJ, Chapman JP, Raulin ML. Body image aberration in schizophrenia. J Abnorm Psychol. 1978;87:399-407.

25. Chapman LJ, Chapman JP, Kwapil TR, Eckblad M, Zinser MC. Putatively psychosis-prone subjects 10 years later. J Abnorm Psychol. 1994;103(2):171-83.

26. Sheinbaum T, Bedoya E, Ros-Morente A, Kwapil TR, Barrantes-Vidal N. Association between attachment prototypes and schizotypy dimensions in two independent non-clinical samples of Spanish and American young adults. Psychiatry Res. 2013;210(2):408-13.

27. Fonseca-Pedrero E, Paino M, Lemos-Giraldez S, Sierra-Baigrie S, Muniz J. Factor structure and measurement invariance of the Wisconsin Schizotypy scales across gender and age. Span J Psychol. 2010;13(2):941-50.

28. William P, Horan MFG, Ann M. Kring, Keith H. Nuechterlein: does Anhedonia in schizophrenia reflect faulty memory for subjectively experienced emotions? J Abnorm Psychol. 2006;115(3):496-508.

29. Fonseca-Pedrero E, Paino M, Lemos-Giraldez S, Garcia-Cueto E, VillazonGarcia U, Bobes J, Muniz J. Psychometric properties of the revised physical and social Anhedonia scales in non-clinical young adults. The Spanish journal of psychology. 2009;12(2):815-22.

30. Chan RC, Wang Y, Yan C, Zhao Q, McGrath J, Hsi X, Stone WS. A study of trait anhedonia in non-clinical Chinese samples: evidence from the Chapman scales for physical and social Anhedonia. PLoS One. 2012;7(4):e34275.

31. Cihan B, Saka MC, Gonullu I, Ozel Kizil ET, Baskak B, Atbasoglu EC. Exploring the role of social Anhedonia in the positive and negative dimensions of Schizotypy in a non-clinical sample. Noro Psikiyatri Arsivi. 2015;52(3):272-8.

32. Horan WP, Blanchard JJ, Gangestad SW, Kwapil TR. The psychometric detection of schizotypy: do putative schizotypy indicators identify the same latent class? J Abnorm Psychol. 2004;113(3):339-57.

33. Blanchard JJ, Horan WP, Brown SA. Diagnostic differences in social anhedonia: a longitudinal study of schizophrenia and major depressive disorder. J Abnorm Psychol. 2001;110(3):363-71.

34. Kosmadakis CS, Bungener C, Pierson A, Jouvent R, Widlocher D. Translation and validation of the Revised Social Anhedonia Scale. L'Encephale. 1995;21(6):437-43.

35. Blanchard JJ, Mueser KT, Bellack AS. Anhedonia, positive and negative affect, and social functioning in schizophrenia. Schizophr Bull. 1998;24(3):413-24.

36. Loas G. Adaptation and French validation of physical anhedonia scale: PAS (Chapman and Chapman, 1978) L'Encephale 1993, 19(6):639-644.

37. Bailer J, Volz M, Diener C, Rey ER. Reliability and validity of German version of the physical and social anhedonia scales and the perceptual aberration scale. Z Klin Psychol. 2004;33(1):15-23.

38. Burgdorfer $G$, Hautizinger M. Evaluation and revision of scales for measurement of physical and social anhedonia. European archives of psychiatry and Clinical. 1987;236(4):223-9.

39. Miettunen J, Veijola J, Freimer N, Lichtermann D, Peltonen L, Paunio T, Isohanni M, Joukamaa M, Ekelund J. Data on schizotypy and affective scales are gender and education dependent--study in the northern Finland 1966 birth cohort. Psychiatry Res. 2010;178(2):408-13.

40. Chan RC, Gooding DC, Shi HS, Geng FL, Xie DJ, Yang ZY, Liu WH, Wang Y, Yan C, Shi C, et al. Evidence of structural invariance across three groups of Meehlian schizotypes. NPJ Schizophr. 2016:2:16016.

41. Chan RC, Shi HS, Geng FL, Liu WH, Yan C, Wang Y, Gooding DC. The Chapman psychosis-proneness scales: consistency across culture and time. Psychiatry Res. 2015;228(1):143-9. 
42. Reise SP, Horan WP, Blanchard JJ. The challenges of fitting an item response theory model to the social Anhedonia scale. J Pers Assess. 2011;93(3):21324.

43. Leak GK. An examination of the construct validity of the social Anhedonia scale. J Pers Assess. 1991;56(1):84-95.

44. Blanchard JJ, Gangestad SW, Brown SA, Horan WP. Hedonic capacity and schizotypy revisited: a taxometric analysis of social anhedonia. J Abnorm Psychol. 2000;109(1):87-95.

45. Cicero DC, Krieg A, Becker TM, Kerns JG. Evidence for the discriminant validity of the revised social Anhedonia scale from social anxiety. Assessment. 2016;23(5):544-56.

46. Kwapil TR, Barrantes-Vidal N, Silvia PJ. The dimensional structure of the Wisconsin Schizotypy scales: factor identification and construct validity. Schizophr Bull. 2008;34(3):444-57.

47. Smith EA, Bolinskey PK, Guidi JP, Myers KR, Schuder KM, James AV, Hudak DV, Sheets V. Further examination of the factor structure of the Chapman psychosis proneness scales (CPPS). Psychiatry Res. 2016;238:257-63.

48. Gross GM, Mellin J, Silvia PJ, Barrantes-Vidal N, Kwapil TR. Comparing the factor structure of the Wisconsin Schizotypy scales and the schizotypal personality questionnaire. Pers Disord. 2014;5(4):397-405.

49. Gard DE, Gard MG, Kring AM, John OP. Anticipatory and consummatory components of the experience of pleasure: a scale development study. J Res Pers. 2006:40(6):1086-102.

50. Treadway MT, Zald DH. Parsing Anhedonia: translational models of rewardprocessing deficits in psychopathology. Curr Dir Psychol Sci. 2013;22(3):244-9.

51. Horan WP, Green MF, Kring AM, Nuechterlein KH. Does anhedonia in schizophrenia reflect faulty memory for subjectively experienced emotions? J Abnorm Psychol. 2006;115(3):496-508.

52. Leventhal AM, Chasson GS, Tapia E, Miller EK, Pettit JW. Measuring hedonic capacity in depression: a psychometric analysis of three anhedonia scales. J Clin Psychol. 2006;62(12):1545-58.

53. Kline RB. Principles and practice of structural equation modeling. 2nd ed. New York: Guilford; 2005

54. Loehlin JC. Guuttman on factor analysis and group differences: a comment. Multivariate Behav Res. 1992;27(2):235-7.

55. Beck AT, Brown GK, Steer RA. Manual of Beck depression inventory-II. Am Univ Washington dc. 1996;21:88.

56. Beck AT, Brown GK, Steer RA. An inventory for measuring clinical anxiety: psychometric properties. J Consult Clin Psychol. 1988;56(6):893-7.

57. Bernstein DP, Stein JA, Newcomb MD, Walker E, Pogge D, Ahluvalia T. Development and validation of a brief screening version of the childhood trauma questionnaire. Child Abuse Negl. 2003:27:169-90.

58. Bandalos DL. Is Parceling Really Necessary? A Comparison of Results From Item Parceling and Categorical Variable Methodology. Structural Equation Modeling: A Multidisciplinary Journal. 2008;15(2):211-40.

59. Wang MC, Li XM AC, Dai XY, Zhu XZ, Yao SY. The factorial invariance across gender of three well-supported models further evidence for a fifive-factor model of posttraumatic stress disorder. J Nerv Ment Dis. 2013;201:145-52.

60. Hu L, Bentler PM. Cutoff criteria for fit indexes in covariance structure analysis: conventional criteria versus new alternatives. Struct Equ Model, Itidiscip J. 1999;6(1):1-55.

61. Zhao HF, He JY, Yi JY, Yao SQ. Factor structure and measurement invariance across gender groups of the 15-item geriatric depression scale among Chinese elders. Front Psychol. 2019;10:1360.

62. Lamash $\mathrm{L}$, Josman N. Full-information factor analysis of the daily routine and autonomy (DRA) questionnaire among adolescents with autism spectrum disorder. J Adolescence. 2020;79:221-31.

63. Buck B, Lysaker PH. Consummatory and anticipatory anhedonia in schizophrenia: Stability, and associations with emotional distress and social function over six months. 2013;205(1-2):30-35.

64. Loren J. Chapman WSE, and Jean P. Chapman: physical Anhedonia, perceptual aberration, and psychosis pronene. Schizophr Bull. 1980;6(4):639-53.

65. Czeh B, Fuchs E, Wiborg O, Simon M. Animal models of major depression and their clinical implications. Prog Neuro-Psychopharmacol Biol Psychiatry. 2016;64:293-310.

66. Wilson C, Terry AV, Jr. Neurodevelopmental animal models of schizophrenia: role in novel drug discovery and development. Clin Schizophrenia Related Psychoses. 2010;4(2):124-37.

67. Young JW, Zhou X, Geyer MA. Animal models of schizophrenia. Curr Top Behav Neurosci. 2010;4:391-433.
68. Finnell JE, Lombard CM, Padi AR, Moffitt CM, Wilson LB, Wood CS, Wood SK. Physical versus psychological social stress in male rats reveals distinct cardiovascular, inflammatory and behavioral consequences. PLoS one. 2017; 12(2):e0172868

69. Ledo JH, Azevedo EP, Clarke JR, Ribeiro FC, Figueiredo CP, Foguel D, De Felice FG, Ferreira ST. Amyloid-beta oligomers link depressive-like behavior and cognitive deficits in mice. Mol Psychiatry. 2013;18(10):1053-4.

70. Delawalla Z, Barch DM, Fisher Eastep JL, Thomason ES, Hanewinkel MJ, Thompson PA, Csernansky JG. Factors mediating cognitive deficits and psychopathology among siblings of individuals with schizophrenia. Schizophr Bull. 2006:32(3):525-37.

71. Swerdlow NR, Gur RE, Braff DL. Consortium on the genetics of schizophrenia (COGS) assessment of endophenotypes for schizophrenia: an introduction to this special issue of schizophrenia research. Schizophr Res. 2015:163(1-3):9-16.

72. Gur RE, Calkins ME, Gur RC, Horan WP, Nuechterlein KH, Seidman L, Stone WS. The Consortium on the genetics of schizophrenia: neurocognitive endophenotypes. Schizophr bull. 2007;33(1):49-68.

73. Badamasi IM, Lye MS, Ibrahim N, Stanslas J. Genetic endophenotypes for insomnia of major depressive disorder and treatment-induced insomnia. Journal of neural transmission. 2019;126(6):711-22.

74. Bai M, Zhang L, Zhu XZ, Zhang Y, Zhang S, Xue L. Comparison of depressive behaviors induced by three stress paradigms in rats. Physiol Behav. 2014;131:81-6.

75. Bortolon C, Macgregor A, Capdevielle D, Raffard S. Apathy in schizophrenia: A review of neuropsychological and neuroanatomical studies. Neuropsychologia. 2018;118(Pt B):22-33.

76. Gard DE, Kring AM, Gard MG, Horan WP, Green MF. Anhedonia in schizophrenia: distinctions between anticipatory and consummatory pleasure. Schizophr Res. 2007;93(1-3):253-60.

77. Fortunati R, Ossola P, Camerlengo A, Bettini E, De Panfilis C, Tonna M, Maggini C, Marchesi $C$. Anhedonia in schizophrenia: the role of subjective experiences. Compr Psychiatry. 2015;62:152-60.

78. Enneking V, Krussel P, Zaremba D, Dohm K, Grotegerd D, Forster K, Meinert $\mathrm{S}$, et al. Social anhedonia in major depressive disorder: a symptom-specific neuroimaging approach. Neuropsychopharmacol. 2019;44(5):883-9.

79. Shankman SA, Nelson BD, Harrow M, Faull R. Does physical anhedonia play a role in depression? A 20-year longitudinal study. J Affect Disord. 2010; 120(1-3):170-6.

80. Visser KF, Chapman HC, Ruiz I, Raugh IM, Strauss GP: A meta-analysis of selfreported anticipatory and consummatory pleasure in the schizophreniaspectrum. J Psychiatr Res. 2020;21:68-81.

\section{Publisher's Note}

Springer Nature remains neutral with regard to jurisdictional claims in published maps and institutional affiliations.
Ready to submit your research? Choose BMC and benefit from:

- fast, convenient online submission

- thorough peer review by experienced researchers in your field

- rapid publication on acceptance

- support for research data, including large and complex data types

- gold Open Access which fosters wider collaboration and increased citations

- maximum visibility for your research: over $100 \mathrm{M}$ website views per year

At $\mathrm{BMC}$, research is always in progress.

Learn more biomedcentral.com/submission 